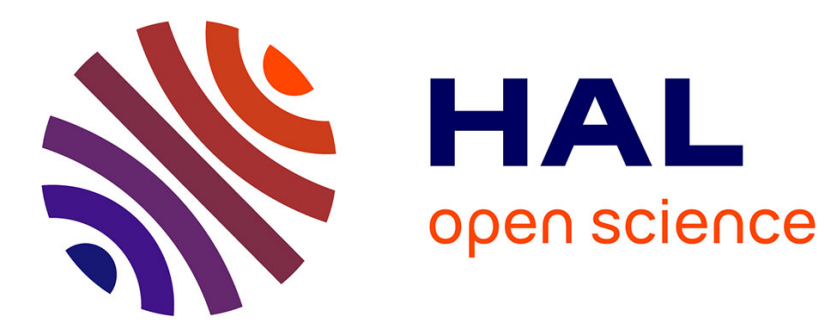

\title{
Multiple Base Stations Diversity for UNB Systems: Theoretical Analysis and Performances
}

\author{
Yuqi Mo, Claire Goursaud, Jean-Marie Gorce
}

\section{To cite this version:}

Yuqi Mo, Claire Goursaud, Jean-Marie Gorce. Multiple Base Stations Diversity for UNB Systems: Theoretical Analysis and Performances. ISNCC 2018 - International Symposium on Networks, Computers and Communications, Jun 2018, Rome, Italy. pp.1-6. hal-01887619

\section{HAL Id: hal-01887619 \\ https://hal.inria.fr/hal-01887619}

Submitted on 5 Oct 2018

HAL is a multi-disciplinary open access archive for the deposit and dissemination of scientific research documents, whether they are published or not. The documents may come from teaching and research institutions in France or abroad, or from public or private research centers.
L'archive ouverte pluridisciplinaire HAL, est destinée au dépôt et à la diffusion de documents scientifiques de niveau recherche, publiés ou non, émanant des établissements d'enseignement et de recherche français ou étrangers, des laboratoires publics ou privés. 


\title{
Multiple Base Stations Diversity for UNB Systems: Theoretical Analysis and Performances
}

\author{
Yuqi $\mathrm{MO}^{* \dagger}$, Claire GOURSAUD*, and Jean-Marie GORCE* \\ ${ }^{*}$ CITI Labs, INSA-Lyon, Villeurbanne, France \\ ${ }^{\dagger}$ Sigfox Company, Building E-volution, Labège, France \\ Email: mo.yuqi@insa-lyon.fr; claire.goursaud@insa-lyon.fr; \\ jean-marie.gorce@insa-lyon.fr;
}

\begin{abstract}
UNB (Ultra Narrow Band) is one of the technologies dedicated to low-power wide-area communication for IoT, currently exploited by SigFox. The specificity of UNB is the Aloha-type channel access scheme, both asynchronized in time and frequency domain. This randomness can cause partial spectral interference. In this paper, we take advantage of the spatial diversity of multiple base stations to improve the UNB performance, by using selection combining. In the presence of pathloss and spectral randomness of $\mathrm{UNB}$, the channels are considered correlated. A theoretical analysis of outage probability is demonstrated by considering this correlation, for the case of 2 base stations. This methodology of probability computing can be extended to general $K$ BSs. The diversity of multiple receivers is proved to be beneficial in enhancing the performance of UNB networks. This gain is shown to be related to the density of the base stations, as well as the distance between each of them.
\end{abstract}

Keywords-IoT, Sigfox, UNB, spectral randomness, multiple base stations, spatial diversity, selection combining, outage probability.

\section{INTRODUCTION}

IoT (Internet of Things) has been gaining a lot of attention in recent years. It is discussed in research, in industry and in every daily life. As the need of connecting objects grows, the challenges for IoT networks grows as well. From the base stations' side, it requires the scalability to connect a large number of objects, and the capability of treating enormous simultaneous transmissions. From the devices' side, the energy consumption and cost are big issues, as most of the devices are battery-based.

Aside from current and forecast traditional cellular networks, LPWAN (Low Power Wide Area Network) have emerged to meet these specific demands of IoT [1]. These new dedicated transmission technologies include CSS (known as LoRa) [2], RPMA (developed by Ingenu) [3], and UNB (known as SigFox) [4], which all operate in the unlicensed ISM band. UNB (Ultra Narrow Band) is expected to be more power efficient than the others, and it allows a longer transmission distance [5] [6]. This technology has already been deployed in Sigfox network around the world, however, even though the point to point link is well-known, the capacity with multiple base stations remains not yet evaluated. Hence, we chose to study UNB, and focus on the uplink of such system.

\section{A. Ultra Narrow Band}

The specificity of UNB is that data is transmitted with an extremely narrow signal band (typically 100-200 Hz), compared to the operating band (typically $200 \mathrm{kHz}-1 \mathrm{MHz}$ ). As a consequence, contrarily to classical systems, the inherent oscillator jitter is not negligible anymore and prevents from allocating disjoint frequency channel to users or set of users. Thus, in practice, transmissions are performed at any carrier frequency within the operating band. The channel access scheme of UNB is R-FTMA (Random Frequency Time Multiple Access), which is an Aloha-type scheme, but both unconstrained in frequency and asynchronous in time domain. On one hand, this grant-free access saves the headers for channel reservation. But, on the other hand, it may lead to partial interference.

The interference pattern of UNB is analyzed in [7]. It was proved to be related to the frequency spacing between nodes' carrier frequencies. In addition, the uplink capacity of UNB is analyzed in [8]. However, all these works are only focused on the one BS case (Base Station).

\section{B. Spatial Diversity in Cellular Networks}

In most cellular systems, one BS is supposed to serve devices in a specific service area [9]. Nonetheless, when devices send data to their intended BS, they are also captured by adjacent BSs. Thus, a BS actually receives the sum of its useful information, the contribution of devices intended to neighboring $\mathrm{BSs}$ and noise. If the BSs independently decode the signal, the neighbor devices are seen as interferers. Moreover, each BS gets a different point of view of the transmitted signal. Indeed, they are not located at the same distance from the transmitting devices, so the transmitted signals experience diverse channel conditions to reach each BS. Similarly, they do not experience the same interference pattern. It is possible that lost packets from a given BS can be properly decoded at another BS. Therefore, taking advantage of the diversity of multiple BS can be beneficial to improve the system performance.

The earliest works about multiple BSs consider them independent and non-cooperative [10]. As packets experience different channel conditions, when one packet fails to be seized by one BS, it is still possible to be captured by another one. The authors in [11] analyzed a cellular network's performance 
of non-cooperative receivers, for slotted ALOHA case. A recent theoretical analysis of the throughput for multiple BSs with perfect power control is presented in [12].

Besides, the interaction and cooperation among BSs also came on the scene, by using different combining and interference cancellation technologies. This cooperation is usually operated at the back-haul, where all BSs transmit their perceived signals. This data center collects information from each BS and treats them jointly. This architecture can be referred to Cloud-RAN (radio access network) [13].

Combining technology, in a general sense, aims at combining the signals each $\mathrm{BS}$ receives, even if none of them can decode it. In this case, the desired user's contribution is constructively added, while interference and noise are averaged, leading to a significant increase in the SINR. Thus, this signal is more likely to be decoded. Maximum ratio combining (MRC) and optimum combining are often analyzed for multiple BSs, by using stochastic geometry as tools [14] [15]. The most frequent assumption is that each node is attached to its nearest BS. With the use of Poisson Point Process, the BS is supposed to be within the Veronoi cell of the user [14]. However, this hypothesis is idealized, and does not fit in our network where nodes are not attached to one specific BS.

Selection combining, as one the combining technologies, selects the strongest signal (of the same message) perceived by all the BSs. Once this strongest signal managed to be decoded, the issued message is considered successfully received. The majority of the existing works about selection combining neglect the dependence among the channels, and thus assume that they are independent [16]. Indeed, with the presence of fast fading, this correlation is weakened, but it does not disappear. The assumption of independent channels is only valid for certain node densities [16] [17]. In this paper, we consider only the pathloss, which faces directly the dependence among the links between the issued node and each BS.

\section{Motivation and Contributions}

All the above-mentioned works are based on traditional channel access where there is either no collision (as transmissions are performed on different orthogonal channels), or total overlap in the frequency domain. However, due to the continuous selection of carrier frequency in UNB networks, partial frequency overlapping has to be considered. To the best of our knowledge, no such analytical studies of multiple BSs for R-FTMA has been conducted.

Therefore, the novelty of this work is to analyze the spatial diversity of multiple BSs for a UNB system, by taking into account the spatial correlation between the received signals. The main contribution is to provide a closed-form theoretical expression of the performance for 2 BSs case with selection combining, as well as to promote this methodology to general $K$ BSs.

The rest of this paper is organized as follows : Section II gives the modeling and assumptions. Section III provides the
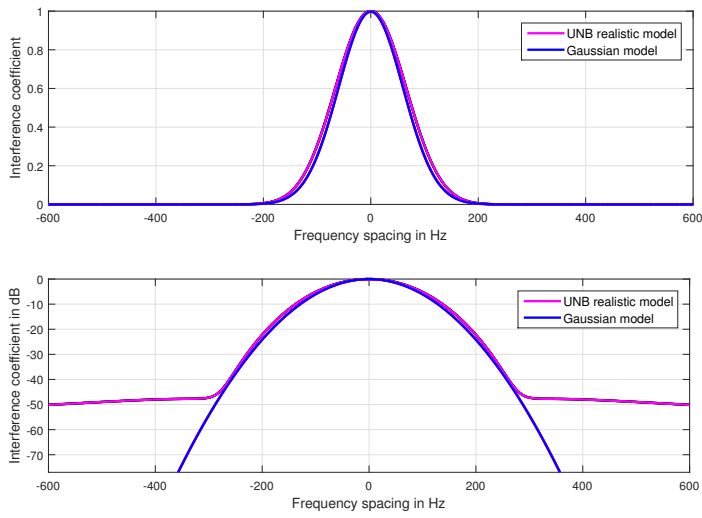

Fig. 1. Behavior of the interference coefficient vs the frequency spacing $\Delta f$ between the desired node and the interfering node

theoretical analysis of the performance for 2 BSs case. The numerical results of $K$ BSs and the validation of theory are given in Section IV. Finally, Section V concludes the paper.

\section{Modeling AND Hypothesis}

In this work, we consider the uplink case, where nodes transmit data to BSs by using the UNB technology. The emission of data is not continuous in time. Nodes are distributed randomly and uniformly in a wide area with a node density $\lambda_{t}$. Due to the very small duty cycle of the considered nodes, we prefer to use the active nodes density $\lambda$ (nodes $/ \mathrm{m}^{2}$ ), which is considered stable at the observed moment. One may note that $\lambda<<\lambda_{t}$. All transmissions are performed with the same emission power and antenna gain. The considered propagation model is free space. Hence the received power of each packet varies, only depending on the respective distance between the node and the issued BS.

BSs are assumed to be powered all the time. Each BS transmits its received messages to the back-haul via wired networks, where the process of selection combining happens.

Nodes broadcast their small data packets to potential multiple BSs with UNB technology. Since nodes select their carrier frequency randomly and independently, a packet may be lost at a BS due to collisions when simultaneous transmissions happen. We only consider in this paper the collisions due to a unique interfering node at the observed moment, from one BS's point of view. Indeed, this is the most frequent case due to the very limited signal bandwidth of UNB [7]. Besides, we neglect the noise power, because it is much lower compared to the interference power level.

One may note that the interference level depends not only on the interferer's received power at the $\mathrm{BS}$, but also on $\Delta f$ the frequency spacing between the desired node and the interfering node. The interference coefficient for realistic filters (as used in SigFox's network) is plotted on Fig. 1. For the theoretical analysis, we have approximated the interference level by a zero-mean Gaussian function [18]:

$$
\beta(\Delta f)=\frac{150}{\sigma \sqrt{2 \pi}} \exp ^{\frac{-\Delta f^{2}}{2 \sigma^{2}}}
$$


with $\sigma=60$ for a $100 \mathrm{bit} / \mathrm{s}$ transmission [7].

\section{THEORETICAL ANALYSIS}

In this section, we derive a closed-form $O P$ (outage probability) expression for $2 \mathrm{BSs}$, with selection combining and the spectral randomness of UNB. Indeed, two BSs is the atomic pattern for any multiple BSs case, which can give us an intuition of their behaviors. Then we extend the methodology to $K \mathrm{BSs}$ in the next section.

Without loss of generality, we consider that the 2 BSs are vertically centered while their horizontal position depends on the relative distance $d$ between each other, as shown on Fig. 2 .

We focus on the performance of one targeted desired node, which is within the communication ranges of the $2 \mathrm{BSs}$, at a known position (not necessarily aligned with the $2 \mathrm{BSs}$ ). All other nodes are potential interfering nodes, and their positions are random. We define $r_{x i}$ (resp. $r_{y i}$ ) (for $i \in\{1,2\}$ ) as the distance between the desired node $x$ (resp. the interfering node $y)$ and $B S_{i}$.

Due to different interference contributions, $B S_{1}$ and $B S_{2}$ perceive the desired user's signal with different SIR. Taking example of $B S_{1}$, the $S I R_{1}$ can be expressed as:

$$
S I R_{1}=\frac{P_{e}\left(\frac{r_{0}}{r_{x 1}}\right)^{2}}{P_{e}\left(\frac{r_{0}}{r_{y 1}}\right)^{2} \beta(\Delta f)}=\left(\frac{r_{y 1}}{r_{x 1}}\right)^{2} \frac{1}{\beta(\Delta f)}
$$

with $P_{e}$ the power at the reference distance $r_{0}$.

In the single $\mathrm{BS}$ case, $O P$ represents the probability that the SIR (Signal to Interference Ratio) of the desired node is lower than a targeted threshold $\gamma^{*}$. As a consequence, and as shown in [8], for the single BS case, a failure is observed when an interferer, whose carrier frequency differs from the desired user of $\Delta f$, falls within a circle of radius $r_{x 1} \sqrt{\gamma^{*} \beta(\Delta f)}$.

Similarly, in the 2 BSs case, data is lost when the SIRs perceived by both BSs are lower than $\gamma^{*}$. Thus $O P$ becomes:

$$
\begin{aligned}
O P & =\mathbb{P}\left(S I R_{1}<\gamma^{*} \cap S I R_{2}<\gamma^{*}\right) \\
& =\mathbb{P}\left(r_{y_{1} 1}<r_{x 1} \sqrt{\gamma^{*} \beta(\Delta f)} \cap r_{y_{2} 2}<r_{x 2} \sqrt{\gamma^{*} \beta(\Delta f)}\right) \\
& =\mathbb{P}\left(r_{y_{1} 1}<R_{x 1}(\Delta f) \cap r_{y_{2} 2}<R_{x 2}(\Delta f)\right)
\end{aligned}
$$

with $R_{x 1}(\Delta f)=r_{x 1} \sqrt{\gamma^{*} \beta(\Delta f)}$ and $R_{x 2}(\Delta f)=$ $r_{x 2} \sqrt{\gamma^{*} \beta(\Delta f)}$. One may note that $R_{x 1}(\Delta f)$ and $R_{x 2}(\Delta f)$ will be written as $R_{x 1}$ and $R_{x 2}$ in the rest of the paper.

As mentioned in Section I, since the channels for both BSs are correlated, we can not simply express $O P=O P_{1} \cdot O P_{2}$. This correlation comes from the fact that there might be one common interferer for both BSs. Thus according to the interferer's location, $O P$ in (3) can be divided into two cases:

1) Common interferer: The same interferer $y=y_{1}=y_{2}$ leads to error in both BSs. In this case, $O P$ is obtained by computing the probability that $y$ falls into the intersection of two circles. The first circle is centered on $B S_{1}$, with a $R_{x 1}$ radius (in blue on Fig. 2), while the second one is centered on $B S_{2}$ with a $R_{x 2}$ radius (in orange). We call this common interfering area as $S c$, whose surface is $s_{c}$. One may note that this area depends on the value of $\Delta f\left(\gamma^{*}\right.$ is predefined). For example, the light blue area in Fig. 2(a) is the non-null intersection $S c$ when $\Delta f=0$, while this area is empty in Fig. 2(b) when $\Delta f=150 \mathrm{~Hz}$.
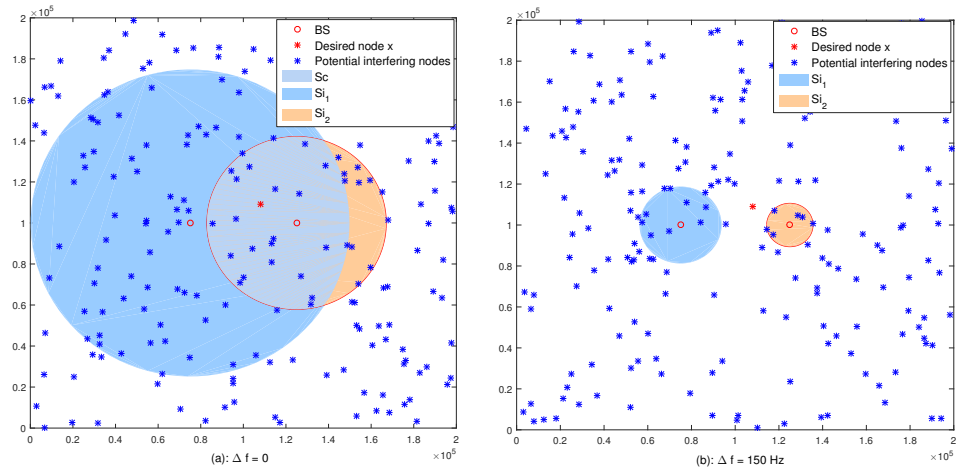

Fig. 2. Topology of the network of (a) $\Delta f=0$ and (b) $\Delta f=150 \mathrm{~Hz}$. $S_{c}$ is the common interfering area, $S i_{1}$ is the interfering circle of $B S_{1}$, and $S i_{2}$ is the interfering circle of $B S_{2}$

2) Distinct interferers: The interferers are different for the $2 \mathrm{BSs}$, which means that there is one specific interferer for each BS. In this case, $O P$ is the probability that the interferer $y_{1}$ falls into the interfering area of BS1 but not inside the common interfering area (i.e. $y_{1}$ is in the blue area $S i_{1}$ in Fig. 2(a) which interferes inside $B S_{1}$ ), while at the same time, another interferer $y_{2}$ falls into $S i_{2}$ (i.e. the orange area).

Therefore, by combining these two disjoints cases, $O P$ becomes:

$O P=\mathbb{P}($ node $y \in S c)+\mathbb{P}\left(\right.$ node $\left.y_{1} \in S i_{1}\right) \cdot \mathbb{P}\left(\right.$ node $\left.y_{2} \in S i_{2}\right)$

Firstly, we focus on the case III-1 (Common interferer). This is the probability that one node in the the considered area falls into the common interfering area $S c$. For the 2 users case:

$\mathbb{P}($ node $y \in S c)=s_{c} \cdot \lambda=\lambda \cdot \int_{0}^{B} s_{c}(\Delta f) \cdot P(\Delta f) \mathrm{d} \Delta f$

with $\lambda$ the node density in nodes $/ \mathrm{m}^{2}$, and $P(\Delta f)=$ $\frac{2}{B}\left(1-\frac{\Delta f}{B}\right)$ as nodes select their carrier frequency randomly within $B W$.

We then compute the common interfering area's surface $s_{c}$. We define $d$ as the distance between two BSs, and use it as an indicator to determine if there is an overlap between the two circles [19]. There are 3 cases for the intersection of two circles, depending on $d$ and both radius $R_{x_{1}}, R_{x_{2}}$, as reported in (6). For the first case, there is a partial overlap between the two circles; for the second case, the two circles are disjoint or too far away, thus no overlap; for the third case, one circle is contained entirely within the other. 
$s_{c}(\Delta f)= \begin{cases}R_{x 1}^{2} \arccos \left(\frac{R_{x 1}^{2}-R_{x 2}^{2}+d^{2}}{2 d R_{x 1}}\right)+R_{x 2}^{2} \arccos \left(\frac{R_{x 2}^{2}-R_{x 2}^{2}+d^{2}}{2 d R_{x 2}}\right)-\frac{\sqrt{\left(\left(R_{x 1}+R_{x 2}\right)^{2}-d^{2}\right) \cdot\left(d^{2}-\left(R_{x 1}-R_{x 2}\right)^{2}\right)}}{2} & \text { if }\left|R_{x 1}-R_{x 2}\right|<d<R_{x 1}+R_{x 2} \\ 0 & \text { if } d \geq R_{x 1}+R_{x 2} \\ \pi \min \left(R_{x 1}, R_{x 2}\right)^{2} & \text { elsewhere }\end{cases}$

Thus the expression of (5) for 2 users in the 2 BS case, can be further written as:

$$
\begin{aligned}
& \mathbb{P}(\text { node } y \in S c)=\int_{0}^{b_{1}}\left(\pi \min \left(R_{x 1}, R_{x 2}\right)^{2} \cdot \lambda \mid \Delta f\right) \cdot P(\Delta f) \mathrm{d} \Delta f \\
& +\int_{b_{1}}^{b_{2}}\left(R_{x 1}^{2} \arccos \left(\frac{R_{x 1}^{2}-R_{x 2}^{2}+d^{2}}{2 d R_{x 1}}\right) \cdot \lambda \mid \Delta f\right) \cdot P(\Delta f) \mathrm{d} \Delta f \\
& +\int_{b_{1}}^{b_{2}}\left(\left(R_{x 2}^{2} \arccos \left(\frac{R_{x 2}^{2}-R_{x 1}^{2}+d^{2}}{2 d R_{x 2}}\right)-\frac{\sqrt{t}}{2}\right) \cdot \lambda \mid \Delta f\right) \cdot P(\Delta f) \mathrm{d} \Delta f
\end{aligned}
$$

with the integral edges defined as following:

$$
\begin{aligned}
& b_{1}=\min \left(\beta^{-1}\left(\frac{d^{2}}{\left(r_{x 1}-r_{x 2}\right)^{2} \gamma^{*}}\right), B\right) \\
& b_{2}=\min \left(\beta^{-1}\left(\frac{d^{2}}{\left(r_{x 1}+r_{x 2}\right)^{2} \gamma^{*}}\right), B\right)
\end{aligned}
$$

We note that $b_{1}$ and $b_{2}$ only exist when both $\frac{d^{2}}{\left(r_{x 1}-r_{x 2}\right)^{2} \gamma^{*}}$ and $\frac{d^{2}}{\left(r_{x 1}+r_{x 2}\right)^{2} \gamma^{*}}$ are in the range of $[0,1]$, otherwise the intersection $S c$ is empty, such as Fig. 2(b).

Secondly, we compute for the case III-2 (distinct interferers). $B S_{1}$ can not decode the desired packet when:

$$
\begin{aligned}
& \mathbb{P}\left(\text { node } y_{1} \in S i_{1}\right) \\
& =s_{i_{1}} \cdot \lambda=\left(\pi R_{x 1}{ }^{2}-s_{c}\right) \cdot \lambda \\
& =\int_{0}^{B}\left(\pi R_{x 1}{ }^{2} \lambda \mid \Delta f\right) \cdot P(\Delta f) \mathrm{d} \Delta f-\int_{0}^{B}\left(s_{c} \cdot \lambda \mid \Delta f\right) \cdot P(\Delta f) \mathrm{d} \Delta f
\end{aligned}
$$

where the second term is already computed in (7). The first term represents in fact the $O P$ when there is only one BS, whose calculation steps are demonstrated in [8], and is given by:

$$
\begin{aligned}
& \int_{0}^{B}\left(\pi R_{x 1}{ }^{2} \lambda \mid \Delta f\right) \cdot P(\Delta f) \mathrm{d} \Delta f \\
& \left.=\frac{300 \pi r_{x 1}{ }^{2} \gamma^{*} \lambda}{\sigma \sqrt{2 \pi} B} \cdot\left(\frac{\sqrt{\sigma^{2} \pi}}{\sqrt{2}} \operatorname{erf}\left(\frac{B}{\sqrt{2 \sigma^{2}}}\right)+\frac{\sigma^{2}}{B}\left(\exp \left(\frac{-B^{2}}{2 \sigma^{2}}\right)-1\right)\right)\right)
\end{aligned}
$$

We can have the expression of $\mathbb{P}\left(\right.$ node $\left.y_{2} \in S i_{2}\right)$ by using the same method:

$$
\begin{aligned}
& \mathbb{P}\left(\text { node } y_{2} \in S i_{2}\right) \\
& =\int_{0}^{B}\left(\pi R_{x 2}{ }^{2} \lambda \mid \Delta f\right) \cdot P(\Delta f) \mathrm{d} \Delta f-\int_{0}^{B}\left(s_{c} \cdot \lambda \mid \Delta f\right) \cdot P(\Delta f) \mathrm{d} \Delta f
\end{aligned}
$$

with the first term similar to (10), and the second term in (7).

We now extend the $O P$ to more users' case. The number of active nodes $N$ depends on the node density $\lambda$ and the considered surface, and it is assumed stable at the observed moment. We have thus $N$ nodes (including the desired node) transmitting simultaneously. With the hypothesis of only one interferer, $O P$ becomes the probability that one out of the $N-1$ nodes enters the common interfering area $s_{c}$, in addition to the probability that two out of $N-1$ nodes enter separately into $S_{i_{1}}$ and $S_{i_{2}}$. Therefore, the final expression of $O P$ for 2 BSs, and $N$ users is:

$$
\begin{aligned}
O P= & \mathbb{P}(\text { node } y \in S c)\left(\begin{array}{c}
N-1 \\
1
\end{array}\right) \\
& +\mathbb{P}\left(\text { node } y_{1} \in S i_{1}\right) \cdot \mathbb{P}\left(\text { node } y_{2} \in S i_{2}\right)\left(\begin{array}{c}
N-1 \\
2
\end{array}\right)
\end{aligned}
$$

with the three issued probabilities presented in (7), (9) and (11).

This derivation of $O P$ gives an intuition for $K(K>2)$ $\mathrm{BSs}$, where we transform $O P$ into the probability that one or several nodes fall into certain areas. And the surface of these areas changes depending on the frequency spacing. The complete derivation for $K \mathrm{BSs}$ is not presented here because of its complexity, but should be deduced by using the same methodology shown above.

\section{Numerical RESUlts AND Exploitation}

In this section, we present the simulation results comparing with the theoretical ones of (12) for 2 BSs. Then we exploit them to measure the benefits two BSs can bring compared to the single BS case. At the end, we present numerical results for the case with $K$ BSs. We note that these Monte-Carlo simulations are performed with Matlab.

In the expression of OP (12), the considered parameters are: the relative distance between the desired node and each BS $r_{x 1}, r_{x 2}$, the distance between two BSs $d$, the node density $\lambda$, the total bandwidth $B$, the number of active nodes $N$ and the SIR threshold $\gamma^{*}$. Simulations are performed by varying these parameters.

\section{A. Validation for $2 \mathrm{BSs}$}

We start by validating the theory for 2 BSs. As illustrated in Fig. 3-4, we have the outage probability for different node density $\lambda$ and total bandwidth $B W$. We compare the $O P$ obtained from (12) and from simulations.

In order to validate the theoretical expression accuracy, we have run simulations by using the realistic Sigfox's interference model (as presented in Fig. 1). We can note that the theory (in solid lines) coincide well with the the simulations results (in points). Such behaviors were also obtained when varying other parameters but are not presented here. The theoretical $O P$ in (12) is hence validated.

At the same time, we can observe on Fig. 3-4 the same behavior of outage probability than the case of one BS in [8]. As the node density $\lambda$ rises, the number of interferers increases accordingly, the $O P$ thus rises up. However, $O P$ declines when the total bandwidth $B W$ increases. This is because when 


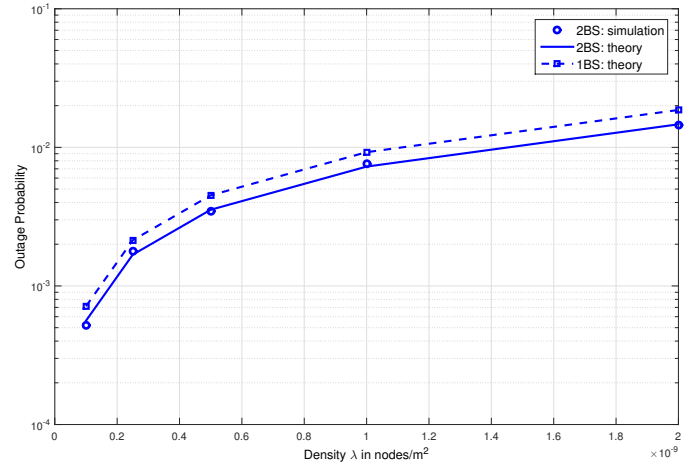

Fig. 3. OP vs $\lambda$, distance between $2 \mathrm{BS} d=5 \mathrm{~km}$, distance between desired node and both BSs $\left(r_{x_{1}}, r_{x_{2}}\right)=(18,22) \mathrm{km}, B=96 \mathrm{kHz}, \gamma^{*}=6.8 \mathrm{~dB}$.

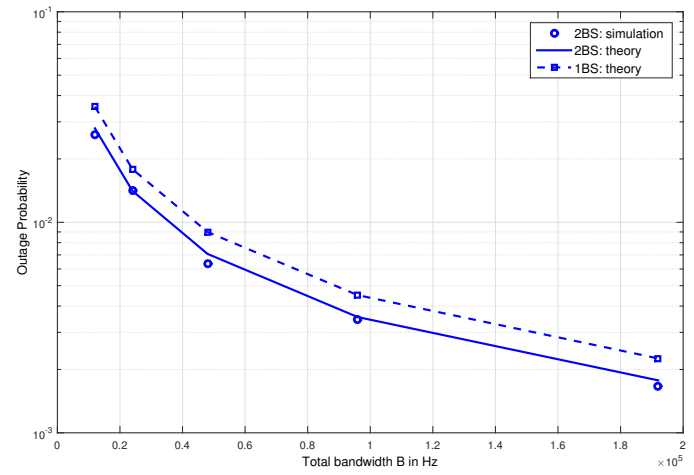

Fig. 4. OP vs $B$, the active node number $N=20$, distance between 2 BS $d=5 \mathrm{~km}$, distance between desired node and both BSs $\left(r_{x_{1}}, r_{x_{2}}\right)=$ $(18,22) \mathrm{km}, \lambda=5 \times 10^{-10}$ nodes $/ \mathrm{m}^{2}, \gamma^{*}=6.8 \mathrm{~dB}$.

the frequency resource becomes larger, the chance that the desired signal gets interfered becomes lower.

\section{B. Exploitation for $2 \mathrm{BSs}$}

We now exploit the theoretical expression in (12) to observe how specific parameters of the $2 \mathrm{BSs}$ case impact $O P$, and the benefits of two BSs compared to the single BS case.

We have firstly varied the distance between two BSs $d$, by maintaining the position of the desired node fixed, as shown in Fig. 5. Consequently, the distances between the desired node and both BSs are varying. We observe that when the two BSs drift apart, $O P$ first drops off and then rises. This is due to the fact that the intersection's surface changes, as illustrated on Fig. 6 which shows the variation of $S c$ as $d$ increases. Accordingly, the number of interferers included in the interfering area differs, which makes the $O P$ vary. When $d$ is small the desired node is close to the BSs. Thus $R_{x 1}$ and $R_{x 2}$ are small but the circles are almost perfectly overlapping. Thus, any node interferes at $B S_{1}$ is also interfering at $B S_{2}$. When $d$ increases, the circles are parted, so the overlap diminishes and $S c$ decreases. However, when $d$ exceeds a certain value, the increase of $d$ leads to the growth of circle radius much sharper than the overlapping reduction. Thus $S c$ increases. Therefore, we can identify the optimal point, such

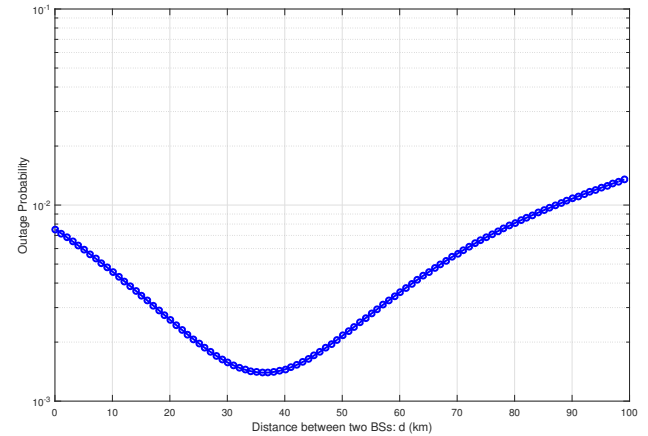

Fig. 5. OP vs the distance between two BSs $d$, the active node number $N=200$, the desired node is fixed, $\lambda=8 \times 10^{-10}$ nodes $/ \mathrm{m}^{2}, B=96$ $\mathrm{kHz}, \gamma^{*}=6.8 \mathrm{~dB}$

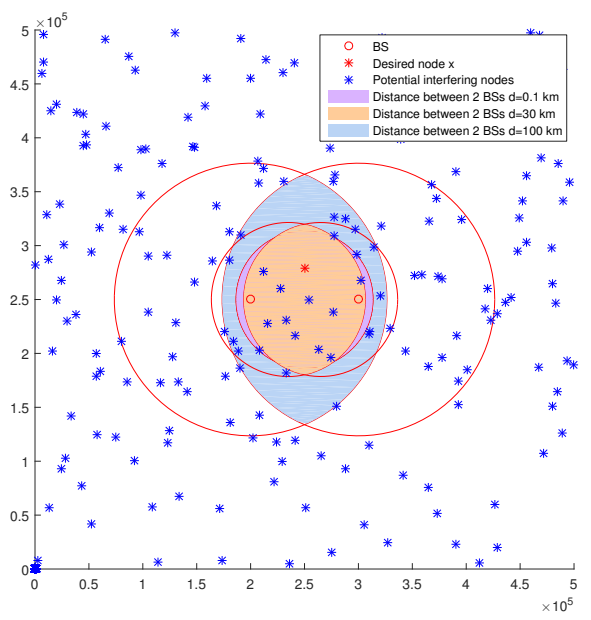

Fig. 6. The intersection $S c$ vs different distance between two BSs $d(\mathrm{~km})$

that for a fixed desired node, there exists a distance $d$ which delivers a lowest $O P$.

In a second step, we focus on the improvement brought by the use of a second BS. To quantify such improvement, we define $\frac{O P_{1 B S}}{O P_{2 B S}}$ as the gain of two BSs. The higher is the gain, the better is the improvement. One may note that we consider in this paper only nodes that are in the reception capabilities of the $2 \mathrm{BSs}$, as the others will not benefit from the second BS. To evaluate this gain, we have plotted $O P$ of a single BS (10) on Fig. 3-4, in dotted lines. We can verify that taking advantage of 2 BSs can improve the network performance, compared to only one BS's case. Indeed, as there is no cell planning among the BSs, and as the nodes broadcast their messages, they can be received by any BS at reach. Thus, the second BS does not affect the performance of the first BS, while bringing an additional chance to receive the packet.

In Fig. 7, we have plotted the variation of the gain as a function of the desired node's position, which is defined by the distance between the desired node and both BSs: $r_{x 1}$ and $r_{x 2}$. We can observe that the gain is always higher when $r_{x 1}=r_{x 2}$, compared to other cases. And we note that the highest gain can be achieved when the desired node is located in between 


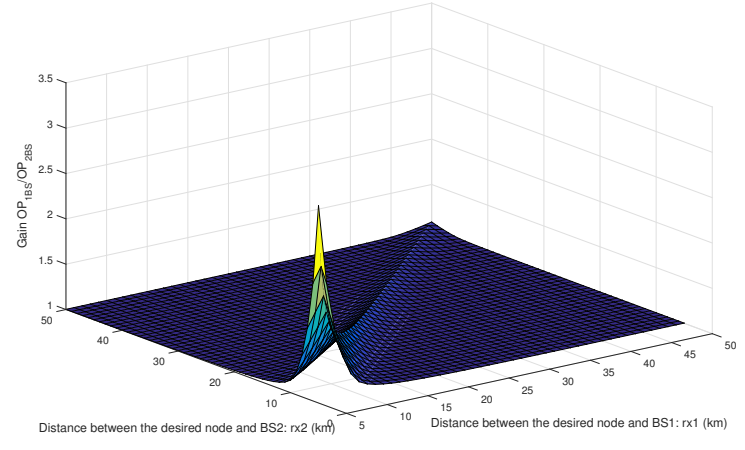

Fig. 7. Gain of two BSs vs the position of desired node presented by $r_{x 1}$ and $r_{x 2}$, the active node number $N=200$, distance between 2 BSs $d=10$ $\mathrm{km}, \lambda=8 \times 10^{-10}$ nodes $/ \mathrm{m}^{2}, B=96 \mathrm{kHz}, \gamma^{*}=6.8 \mathrm{~dB}$.

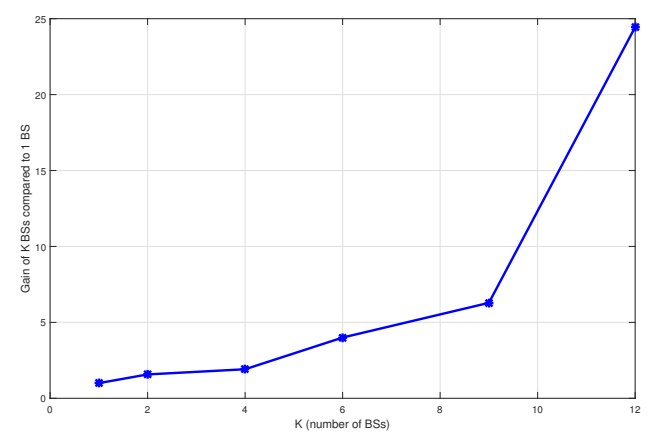

Fig. 8. Gain of multiple BSs vs $K$ the number of BSs, the active node number $N=200$, distance between each BS $d=10 \mathrm{~km}, \lambda=8 \times 10^{-10}$ nodes $/ m^{2}, B W=96 \mathrm{kHz}, \gamma^{*}=6.8 \mathrm{~dB}$.

the two BSs, where its distance to both BSs is equal to $\frac{d}{2}$.

These results can give us an insight about the best gain we can achieve with an additional BS.

\section{Gain of $K B S s$}

Last but not least, we present in Fig. 8 the numerical results for $K \mathrm{BSs}$ (where $K$ is not limited to 2). The locations of BSs form a square lattice, with equal distance $d$ between adjacent BSs. Similarly, we define the gain of $K$ BSs as $\frac{O P_{1 B S}}{O P_{K B S}}$. We observe that when the number of BSs increases, the gain enhances. Indeed, when the BSs becomes denser, their spatial diversity becomes more advantageous. This can also help in the BS deployment and densification.

\section{CONCLuSion}

In this paper, we have derived theoretically the OP (Outage Probability) of a system based on UNB, by taking advantage of the spatial diversity of multiple base stations, and specifically focused on the case of two BSs. We have taken into account the spectral randomness of UNB in the analysis, and we have focused on one targeted desired node. We have brought to light that OP can be transformed into calculating the probability that one node falls into a certain area. And this methodology can be extended for the more general case of multiple BSs. We have validated the theoretical OP by simulations and varying parameters. We have exploited the theory of two BSs to show the highest gain we can achieve from one additional BS. For the general case of $K \mathrm{BSs}$, we have proved that the spatial diversity of multiple BSs is beneficial in improving the networks' performance, and that the benefit is related to the density of BSs. We note that this work has only considered selection combining, and that it can be followed by including more combining technologies in the future.

\section{ACKNOWLEDGMENT}

The authors would like to thank Christophe Fourtet (Sigfox company) for providing realistic UNB network parameters in this study.

\section{REFERENCES}

[1] C. Goursaud and J.-M. Gorce, "Dedicated networks for iot: Phy/mac state of the art and challenges," EAI endorsed transactions on Internet of Things, 2015.

[2] (accessed 2017/07/01). [Online]. Available: http://lora-alliance.org/ What-Is-LoRa/Technology

[3] (accessed 2017/07/01). [Online]. Available: http://www.ingenu.com/

[4] (accessed 2017/07/01). [Online]. Available: http://www.sigfox.com/en/ $\backslash$ \#!/technology

[5] B. Reynders, W. Meert, and S. Pollin, "Range and coexistence analysis of long range unlicensed communication," in 2016 23rd International Conference on Telecommunications (ICT), May 2016, pp. 1-6.

[6] B. Vejlgaard, M. Lauridsen, H. Nguyen, I. Kovács, P. Mogensen, and M. Sørensen, "Coverage and capacity analysis of sigfox, lora, gprs, and nb-iot," in Vehicular Technology Conference. IEEE, 2017.

[7] M.-T. Do, C. Goursaud, and J.-M. Gorce, "Interference modelling and analysis of random fdma scheme in ultra narrowband networks," in AICT 2014, 2014.

[8] Y. Mo, M. T. Do, C. Goursaud, and J.-M. Gorce, "Up-link capacity derivation for ultra-narrow-band iot wireless networks," International Journal of Wireless Information Networks, Jun 2017.

[9] J. Laiho, A. Wacker, and T. Novosad, Radio network planning and optimisation for UMTS. John Wiley \& Sons, 2006.

[10] T. L. Marzetta, "Noncooperative cellular wireless with unlimited numbers of base station antennas," IEEE Transactions on Wireless Communications, vol. 9, no. 11, pp. 3590-3600, 2010.

[11] M. Corson and A. Ephremides, "An analysis of multi-receiver, nonadaptive, slotted aloha with capture for wireless communications in factories," in IEEE INFOCOM 1993 The Conference on Computer Communications, Proceedings. IEEE Comnut. Soc. Press.

[12] A. Munari, F. Clazzer, and G. Liva, "Multi-receiver aloha systems a survey and new results," in 2015 IEEE International Conference on Communication Workshop (ICCW), Jun. 2015, pp. 2108-2114.

[13] A. Checko, H. L. Christiansen, Y. Yan, L. Scolari, G. Kardaras, M. S Berger, and L. Dittmann, "Cloud ran for mobile networks: A technology overview," IEEE Communications Surveys Tutorials, vol. 17, no. 1, pp. 405-426, Firstquarter 2015.

[14] M. D. Renzo and P. Guan, "Stochastic geometry modeling and systemlevel analysis of uplink heterogeneous cellular networks with multiantenna base stations," IEEE Transactions on Communications, vol. 64, no. 6, pp. 2453-2476, Jun. 2016.

[15] T. Bai and R. W. Heath, "Analyzing uplink sinr and rate in massive mimo systems using stochastic geometry," IEEE Transactions on Communications, vol. 64, no. 11, pp. 4592-4606, Nov 2016.

[16] Q. Song, X. Lagrange, and L. Nuaymi, "Evaluation of macro diversity gain in long range aloha networks," IEEE Communications Letters, vol. 21, no. 11, pp. 2472-2475, Nov 2017.

[17] M. Haenggi and R. K. Ganti, "Interference in large wireless networks," Found. Trends Netw., vol. 3, no. 2, pp. 127-248, Feb. 2009. [Online]. Available: http://dx.doi.org/10.1561/1300000015

[18] Y. Mo, C. Goursaud, and J. M. Gorce, "Theoretical analysis of unb-based iot networks with path loss and random spectrum access," in 2016 IEEE 27th Annual International Symposium on Personal, Indoor, and Mobile Radio Communications (PIMRC), Sep. 2016, pp. 1-6.

[19] A. M. J. E. Handricks, The analyst. a journal of pure and applied mathematics, 1876. 